\title{
CHALLENGES TO AND OPPORTUNITIES FOR ESTABLISHING A QUALITATIVE APPROACH TO BUILT ENVIRONMENT RESEARCH IN HIGHER EDUCATION INSTITUTIONS
}

\author{
Nnedinma I Umeokafor \\ Department of Built Environment, Faculty of Architecture, Computing \& Humanities, \\ University of Greenwich \\ Nnedinmaik@hotmail.com \\ Abimbola Windapo, (Department of Construction Economics and Management, \\ University of Cape Town, Cape Town, South Africa)
}

\begin{abstract}
Purpose - There are serious implications for adopting inappropriate research strategies and methods, and this is evident in the Built Environment (BE) given the under adoption of qualitative strategies in some countries. Therefore, based on empirical evidence from Nigeria, the purpose of this study is to examine the challenges to and opportunities for establishing qualitative approach (QA) to $\mathrm{BE}$ research in Higher Education Institutions (HEIs) and develop an improvement framework for QA.

Design/methodology/approach - Academics and research students in the BE research of Nigerian HEIs were interviewed and the data analysed thematically. Based on the findings including recommendations from the respondents, a framework for improving the use of $\mathrm{QA}$ in $\mathrm{BE}$ research was developed and academics evaluated it for workability.

Findings - The study reveals that the challenges to QA in BE research include information constraints, socio-cultural issues and the negative attitudes of senior academics to QA. The opportunities include the realisation for a paradigm shift, the characteristics of the socio-cultural context and features of $\mathrm{BE}$ and the general potentials of QA. The proposed framework encompasses encouraging and providing a platform for international collaboration between academics in developing and developed countries, and preferential treatment for QA. It also enables regulatory and incentive mechanisms which will act as drivers.
\end{abstract}

Practical implications - This study provides stakeholders in academia with knowledge and a detailed guideline for establishing QA to research in the BE.

Originality/value - The study provides a country context-based detailed guide for establishing QA in HEIs BE research towards ensuring that research strategies adopted in research in BE research are fit for purpose, in turn aligned to addressing problems in the society. There is little or no study of this nature in BE.

Keywords: academia, built environment, higher education, qualitative research, sociocultural indicators. 


\section{INTRODUCTION}

Although not absolute, qualitative strategies and methods are mainly of interpretivist or constructivist paradigm involving methods such as unstructured interviews and observations (Saunder et al. 2009). Quantitative strategies and methods are primarily of empiricist, rationalist, and positivist paradigm involving methods such as experiments and statistical survey (Saunder et al. 2009). Interpretivist or constructivist paradigm assumes the ontological position that there is multiple truth in reality, it is subjective and susceptible to the influence of the investigator while the positivist paradigm assumes that reality is concrete, one truth and cannot be affected by the researcher (Saunder et al. 2009; Umeokafor 2015). According to Kheni (2008), the epistemological position of the latter is that only what can be observed is the truth and sound knowledge while the former assumes that sound knowledge is subjective and socially constructed by individuals in the environment. By implication, qualitative strategies and methods provide a thorough understanding of social phenomena, generating theories in some cases.

An alternative paradigm, pragmatism, which underpins mixed methods, compliments any drawbacks of the preceding two paradigms (Morgan 2006). Pragmatism is not biased or committed to a particular paradigm, research strategies and methods. This is because pragmatism assumes that the study problems or questions determine the research strategy or approach adopted in research (Pollack 2007; Robson 2002; Saunders et al. 2009). By implication, if the inappropriate research strategy or approach is adopted, there are grave implications for research and its problem solving aim (AlSehaimi et al. 2013). This forms the premise of the current study.

The implications of adopting inappropriate research strategies and methods are noted, suggested or demonstrated in studies not limited to AlSehaimi et al. (2013), Crossley and Vulliamy (1996), Hughes (2010), and Laryea and Leiringer (2012). For example, the research problems in construction management studies, delay in construction, are addressed with inappropriate research strategies and methods, such as quantitative methods, resulting in impractical recommendations that do not even match the findings hence unfit for purpose (AlSehaimi et al. 2013).

There is evidence in studies such as Crossley and Vulliamy (1996) and Kheni (2008) that developing countries (DCs) provide opportunities that can be exploited in the qualitative approach paradigm to the advantage of research. In particular, the low level of literacy and numeracy in DCs, a social challenge, and the cultural and historical context of DCs provide opportunities to the qualitative paradigm (Crossley \& Vulliamy 1996). This is discussed in detail in the paper.

Notwithstanding the possibilities in the Built Environment (BE) disciplines of Higher Education Institutions (HEIs) in DCs for QA and the implications of adopting inappropriate research strategies and methods for research, authors such as Ejohwomu and Oshodi (2014), Laryea and Leiringer (2012) demonstrate that quantitative approach studies (mainly surveys) dominate DCs' BE research in countries such as Nigeria. Similarly, in developed countries, there is evidence in studies that qualitative strategies and methods, are underrepresented in BE disciplines such as Construction Management, for example, see Carter and Fortune (2004), Danity, Bagilhole, and Neale (1997) Loosemore, Hall, and Dainty (1996), Panas and Pantouvakis (2010). 
Some Authors offer explanations for the underrepresentation of qualitative approach (QA). According to Umeokafor and Windapo (2017), the explanations for the underrepresentation of QA in the BE of Nigeria include socio-cultural and economic issues and the lack of understanding of the potential of QA. More challenges are noted in studies such as Pryor (1998), Bubaker et al. (2005) and Hurst et al. (2015). Although, the preceding can discourage academics and students in the HEIs of DCs from adopting a qualitative approach (QA) to research, the benefits of addressing research problems using an appropriate research approach makes a case for its use.

It is evident that despite the attention to the under-representation of QA to research in the BE, by authors such as AlSehaimi et al. (2013), Danity et al. (1997, 2000), Panas and Pantouvakis (2010) and Umeokafor and Windapo (2017), more needs to be done, at least from the Nigerian perspective, especially because of the potentials of QA to research. Developing a detailed guide for establishing QA in HEIs BE research remains overlooked by authors. A study of this nature will be beneficial to stakeholders in academia with interest in engendering a paradigm shift in BE studies and those keen on ensuring the research is aligned to addressing problems in the society. It will also help in ensuring currency in research. In developing such a framework, there is need to understand the challenges and opportunities of QA to BE research within HEIs in DCs such as Nigeria. Previous studies, such as Bubaker et al. (2005), do not address challenges and opportunities of QA from a BE perspective. The scope of the study by Bubaker et al. (2005) is limited to one research strategy. Besides, the characteristics of the $\mathrm{BE}$, which is unique and complex, make the transferability of the extant knowledge challenging and inappropriate. Also, the opportunities in DCs of QA to research remain underexplored, at least empirically.

Based on the background established so far, using Nigeria as a case, the objectives of the study are:

A. To establish and explain the challenges in qualitative research within Built Environment Disciplines in HEIs.

B. To establish and explain the opportunities for qualitative research within Built Environment Disciplines in HEIs.

C. To establish the views of respondents on the measures for improving the use of QA in research.

D. To consolidate the research findings by developing a framework for improving the underrepresentation of QA to BE research in HEIs.

Importantly, the study strongly argues that adequate research methods or strategies be adopted in research hence 'fit for purpose'. While according to Umeokafor and Windapo (2017), the bias against qualitative methods and analysis is not limited to Nigeria, in this study, it is used as a case study with the BE forming the context of the study. This is because of reasons not limited to 1) DCs are too many and QA such as Case Studies and Action Research is widely used in BE research communities in some Latin American Countries; 2) limiting it to Nigerian HEIs allows the analytical generalisation of the results to Nigerian HEIs and maintain consistency; 3) this is just a first step to further research in this area.

However, some DCs may find the study helpful, as DCs have a lot on common such as culture and methods of construction (cf. Kheni 2008). Nevertheless, borrowing the 
words of the reviewers, for the interest of a wider community of international readers, emphasis is placed on the implications of this study for DCs.

\section{LITERATURE REVIEW}

The contributions of the public and private sectors in research, rationale and implications for research

The significant contributions or role of the government in research in Organisation of Economic Cooperation for Development (OECD) countries such as the US and New Zealand are covered in literature, for example Bernanke (2011), Maloney and Schumer (2010), Narayan (2010) and United Nations Educational, Scientific and Cultural Organization (UNESCO) (2015). UNESCO (2015) demonstrates that the government is the primary funder of basic research in many of the OECD countries covered. In the US, the government funds research with no specific commercial motive as against private firms who fund research mainly for commercial gain (Bernanke 2011; Maloney \& Schumer 2010). By implication, private companies underfund basic research but the government makes up for this (Bernanke 2011; Maloney \& Schumer 2010). Other contributions or supportive roles of the government include creating tax incentives, running research facilities (Bernanke 2011), providing legislation that protects and supports educational or research institutions' contributions or efforts in research (Narayan 2010). Further rationale for the continued expansion of government assistance for research includes keeping pace with technological advances in other countries (Narayan 2010); providing strategic direction and formulating research priorities in HEI, fostering innovation and supporting academic research commercialisation (Narayan 2010). In Nigeria, the contribution of the government may not be the same as in the former countries. While there are government research institutes, there is Tertiary Education Trust Fund (TETFund) established by the Tertiary Education Trust Fund (TETFund) Act, 2011. TETFund has the responsibility of improving, managing and disbursing tax to public tertiary institutions in Nigeria (TETFund 2011: A255). As a result, TETFund finances, among many, conference attendance, research training such as $\mathrm{PhD}$. The oil and gas industry and few state governments and private firms also fund research training and applied research projects, for commercial gain.

\section{Review of previous studies on the representation of QA in BE research in West Africa including Nigeria}

Laryea and Leiringer (2012) in examining a conference series in West Africa from 2009-2011 show that survey research design papers accounted for four times more than other research strategies including some QA research strategies that accounted for nothing. Overall, quantitative approach papers comprised approximately $74 \%$, while the qualitative research approach studies made up just less than $1 \%$ of the papers presented in the conference series. Laryea and Leiringer (2012) conclude that comparing their findings to publications in 25 leading journals in the past 25 years, that the orientation of research methods are similar. Analogously, Ejohwomu and Oshodi (2014) reviewed PhD thesis from 1984-2012 in the Department of Building and Quantity Surveying of seven Major Nigerian HEIs and found that quantitative studies account for at least $66.67 \%$. Furthermore, a treatise on the discourse, Umeokafor and Windapo (2017), found that QA is underrepresented in the BE of Nigeria and offer deep understanding for this and the disposition of academics towards $H \& S$. In addition to reviewing precious studies that demonstrate the 
underrepresentation of QA in BE research of some countries including Nigeria, Umeokafor and Windapo (2017) also reviewed papers presented at a BE international conferences series from 2013-2014 jointly hosted and organised by Ghana, Nigeria, and South Africa. They found that quantitative methods and strategy is dominant 'within all the 93 papers in the two-conference series proceedings' (Umeokafor and Windapo (2017:7). Umeokafor and Windapo (2017) concluded that 'Qualitative research is in its infancy"; "... qualitative techniques are almost non-existent", and that the disposition of the academics is biased towards QA, amenable, among many, QA is to be conducted when convenient.

\section{Selected previous studies on the representation of QA in developed countries' BE research}

Qualitative research in developed countries' Higher Education BE research in general is also of concern in terms of its representation in research publications (Carter \& Fortune 2004; Danity, Bagilhole, \& Neale, 1997; Hyari, El-Mashaleh, \& Rababeh, 2015; Loosemore, Hall, \& Dainty, 1996; Panas \& Pantouvakis 2010). However, the focus of the current study is on Higher Education in DCs, Nigeria in particular. Typically, Loosemore et al. (1996) found that QA based studies covered just $8 \%$ in a high profile construction journal as against quantitative studies that cover $57 \%$. Similarly, Carter and Fortune (2004) reviewed the proceedings of a construction conference series of 2000 and 2001 and postgraduate construction research at HeriotWatt University (HWU) from 2001-2003. In the year 2000, Carter and Fortune (2004) found QA papers to be 6\% and then in 2001 an increase in the number of QA studies to $19 \%$. For HWU research, Carter and Fortune (2004) found QA studies to account for $18 \%$ and quantitative papers just $42 \%$. Recently, reviewing construction productivity papers, Panas and Pantouvakis (2010) found that QA studies account for $10.1 \%$ of the publications in top quality journals from 1999-2009 as against quantitative studies that accounted for $60.7 \%$.

\section{Implications of adopting inappropriate research strategies and methods}

AlSehaimi et al. (2013) examined construction delay studies in developing countries and found that the recommendations do not match the findings of the research, contributing little to addressing the problems to which the studies were aimed to address. Aleshaimi et al. go on to demonstrate that the explanations for this include the poor research strategies and methods (such as statistical survey and questionnaires) adopted which aim to identify and describe the current state of affairs and the knowledge produced - descriptive and explanatory research (2013). Consequently, AlSehaimi et al. (2013) recommended qualitative strategies such as constructivist and action research for solving the problems in construction delay studies in developing countries. This is because these QA strategies enable 'close collaboration with industry partners, ... solving practical problems and generating new knowledge in the form of systems, models, or frameworks' (AlSehaimi et al. 2013: 411). In the same vein, but earlier, Crossley and Vulliamy (1996) note that the results of some studies are unreliable because the research questions were wrongly tackled with inappropriate research strategies and methods. There are also implications for the students regarding the knowledge impacted by the lecturers in that it may be questionable (Hughes 2010; Umeokafor \& Windapo 2017). This is because lecturers that are biased against QA are likely to discourage students (Umeokafor \& Windapo, 2017). 


\section{Challenges in Qualitative Research in HEIs of developing countries: Explanations for the underrepresentation of QA}

The challenges seem not to have significantly transmuted over the years, but there are a few new dimensions to the discourse. Pryor (1998) while discussing action research in West Africa (Ghana) covers issues in qualitative research such as action research not fitting into the activities of lecturers because of lack of resources, time and energy; lecturers in Pryor's work keep waiting for instructions on what to do at the expense of being innovative. Pryor goes on to cover other issues such as resistance to change, as qualitative research may be considered different from what some of the lecturers know or have been taught (1998). In the work of Danity et al. (2000), they cover the 'time-consuming' challenge in QA in general but also analytical software that reduces the time spent on the analysis, making the analysis easier and more transparent.

In the work of Bubaker et al. (2005) based in Libya and Malaysia, they echo the resources and skills issues noted in Pryor's work but expand, covering further challenges that can deter researchers in HEIs of DCs. Bubaker et al. (2005) discuss the high level of skills needed for qualitative research, funding, logistics, cultural and language barriers, gaining access to organisations in terms of the qualitative case study and the high level of bureaucracy when working with organisations. According to them, some of the organisations do not trust researchers, fearing that they will be exposed. Also, if the researcher does not know the right people in the organisation, the research project may be delayed (Bubaker et al. 2005), leading to frustration and despair.

Other authors go on to support Bubaker et al. (2005). In particular, in Uganda, Mugisha Knizek, Kinyanda and Hjelmeland (2011) stress the occasions where: culture and superstitious beliefs hinder or prohibit respondents from providing information. For example, there are beliefs and fear of implications of discussing or revealing any information about the supernatural or a person in authority such as a king or matters such as suicide (Mugisha et al. 2011). Similarly, based on Nigeria, Hurst et al. (2015) note the challenges that diversity in culture (even when there is a common language) creates in designing of data collection instruments such as the interview guide. This is because different cultures have different connotations to meanings of research questions (Hurst et al. 2015). Mugisha et al. (2011) then introduced a new dimension to the discourse in that legal issues make gaining access to study population difficult, while culture (such as restrictions due to gender) hinders gaining rapport with interviewees.

Still in the same year, 2011 but in support of an earlier study, Pryor (1998), Halai (2011) shows that in Pakistan combining teaching and research may be difficult for lecturers especially when the role involves research strategies such as action research. In some cases, the research strategy such as action research may present a complex condition for researchers (Halai 2011) perhaps discouraging them. These same arguments may apply to lecturers in the BE stream of HEIs in DCs.

In support of Bubaker et al. (2005), Hurst et al. (2015) demonstrate that language challenges comes in different ways, for example, during the translation of the meaning of words and their structures in interview recordings from one language to another. Also, the bias against basic research by private firms that emerged in the 
preceding section and limitations to some disclosures remain a significant barrier. The general challenges in QA to research, such as researcher's perceptions, values, beliefs; transferability of findings; validity and reliability are covered in Umeokafor (2015).

\section{Opportunities in HEIs of developing countries for adopting a qualitative research approach}

In addition to tackling the research questions that quantitative methods are unable to tackle and offering deep insight into social phenomena, the qualitative strategies and methods also present, among other things, the following opportunities. Firstly, the historical and cultural context of DCs means that qualitative research will excel therein (Crossley \& Vulliamy 1996), especially as the activities of disciplines in the built environment (BE), such as architecture, are embedded in QA. The examples of cultural context include collectivist cultural dimension, and the ability of local chiefs and heads of extended families to preserve customary laws and command respect (Kheni 2008). The impact of this on research is covered further in this section. The Arts in Africa can inspire architectural designs. What is reality in one social setting may not be the same in another; reality ...is therefore dependent upon cultural assumption (Kheni 2008:33). Secondly, although, the literacy and numeracy level in DCs has improved, it is still low compared to developed countries, and the characteristics of qualitative research present it with the potential of reaching people in the low literacy and numeracy areas (c.f Crossley \& Vulliamy 1996). This can be through QA research methods such as interviews and obser-view. In particular, Umeokafor (2015) demonstrates that obser-view, a qualitative data generation method, can enhance reflection and learning on the part of the researcher and participant during data collection. Thirdly, QA research methods such as observ-view, and interviews have a potential of establishing a relationship and trust between the investigator and the participant and gives the participant a sense of belonging (Umeokafor 2015) perhaps making them reveal vital information. Fourthly, cultural values will be to the advantage of the researcher in that if they are of the same clan or have an association with the clan (Kheni 2008) or speak the same language with respondents, the respondents are more relaxed (Bubaker et al. 2005). However, the respondents may be tempted to say things that would please the investigator.

\section{METHODOLOGY}

The research problem, aim and objectives of the study meet the conditions for adopting QA to research in that the study focuses on complex social phenomena (Isaacs 2014). In other words, it is a 'how' question in which QA excels (Creswell 1998). However, to ensure rigour in the research, some steps were taken. Firstly, the interview guide was piloted on two BE program lecturers and one student in Nigeria and refined before the main data collection. Before this, the interview protocol was tested for its usability in line with the four-phase interview protocol refinement framework in Castillo-Montoya (2016). This entailed 'Ensuring interview questions align with research questions; constructing an inquiry-based conversation, receiving feedback on the interview protocol; and piloting the interview protocol'. In addition to ensuring that the interview protocol was usable, it ensured that no research question had little, excessive or even no interview questions addressing them. It also ensured that the questions were not confrontational to the respondents but ensuring that, where possible, it elicited a discussion between the interviewee and the investigator. 
Secondly, data triangulation (Denzin 1970) involving research students and academics was conducted. Thirdly, peer debriefing was also adopted where the views of the lead author and activities of the research were discussed with peers in the industry and academia. This is in addition to the discussion between the authors.

The data collection process involved two stages (See Figure 1). The first stage involved the interviews (of between 28- 40 minutes) and e-interviews for addressing the objectives A, B and C, and designing the framework. The second stage- the evaluation of the framework designed in the first phase-involved sending the framework to the respondents to comment on its practicality, with email and interview response options, towards a possible refinement of the framework.

Figure 1: Overall research process

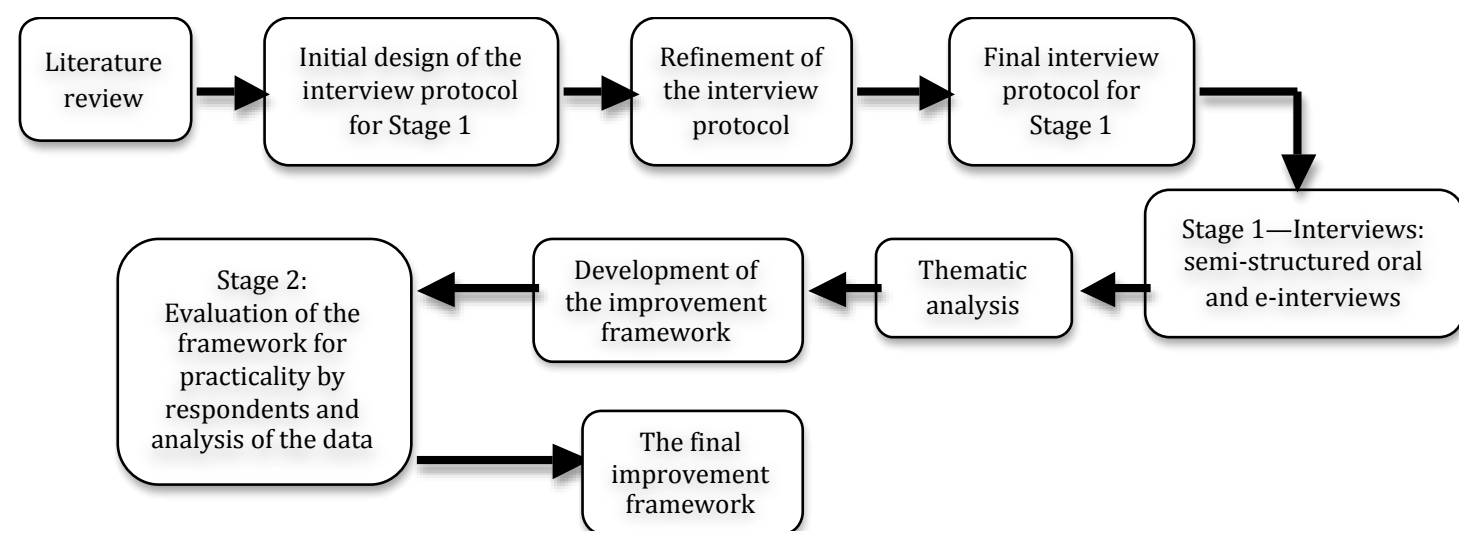

The sample frame for the study constituted of participants of three major international conferences and one doctoral workshop (name purposely withheld). These conferences were held in Nigeria, Ghana and South Africa in 2014, 2015 and 2016, respectively, and the workshop in 2014. One hundred and seventeen academics from the BE disciplines of Nigerian HEIs that attended the conference series and workshop described above were purposely invited to take part in the study. Additionally, nine students from the BE disciplines of Nigerian HEIs were also purposefully invited. The criterion for selecting the academics was that they must be affiliated with a higher or research institution in Nigeria and hold $\mathrm{BE}$ academic roles. If based abroad, they much have studied in Nigeria and worked as a BE Lecturer or researcher in a higher or research institution in Nigeria. This is in addition to having studied BE course(s) at undergraduate and postgraduate levels of which the latter must cover research degree and experience. The gender divide, academic location background, hierarchies in academia, for example lecturer or senior lecturer, and category of higher institution (private and public) were also factored in the sampling. For example, the researchers ensured that no gender was excluded in the sampling. The reason for this is the social nature of the research problem, hence ensuring a realistic representation of the population. In the context of the study, the population of academics in Nigeria was not necessary, as the study did not seek statistical generalisation but a thorough understanding of the social phenomena and generating theories towards developing a framework. Also, the authors of the papers presented at the conferences and workshops are considered to have a good knowledge and representation of the key characteristics of academics in Nigeria and can provide rich information. Bowen (2008) adopted purposeful sampling where information-rich cases were sought. According to Patton (1990), the goal of sampling is ensuring that information-rich 
cases are sampled towards answering the research questions.

Introductory letters and emails were sent to participants, inviting them to take part in the study. The study was explained to them including the two methods of data collection. They were also informed of how the information they provide will be used, assured of anonymity and offered the option of withdrawing from the interview and einterview while it is on-going or within two weeks of data collection. The interviewees were also informed of how and where the data will be stored and that the data will be used solely for research proposes. At the end of the first phase of the study, 17 academics and five students in various BE disciplines of Nigerian HEIs participated in the study while 11 academics participated in the second phase, a total of 33 respondents.

For the first stage of the data collection, the literature review above was used as a framework for designing the semi-structured interviews and the e-interview guides. The guide came in two sections of semi-structured questions. E-interview involves collecting qualitative data from respondents through the exchange of emails (containing questions) between the interviewer and interviewee (Bampton \& Cowton 2002). Carter and Fortune (2004) encourage web-based generated qualitative data, which can be through open-ended questions sent through the Internet just like questionnaires. Carter and Fortune (2004) found that a combination of web and emails as done in Delphi studies provide valid qualitative data than the traditional conference room focus group interview.

The indicative questions of the interviews and e-interviews, among many, addressed the challenges that the respondents have experienced while conducting QA research in the BE in Nigeria. This was aimed at understanding the challenges the respondents have encountered in BE research in Nigeria from their perspective. For example, they were asked to discuss the challenges that they encountered while conducting QA research and how the challenges have influenced or not influenced BE research in Nigeria. Other questions on challenges were then narrowed down to specific challenges that were identified during literature review such as 'considering the human and financial resources, how QA research fits into the academic and research activities of the academics'. Further questions then centred on the opportunities in Nigeria's HEIs for QA to research in the BE. These were aimed at understanding the perceptions and experiences of the academics regarding the opportunities in Nigeria for QA to research in the BE. The final questions sought the views of the respondents on the ways to improve the under-representation of QA to research in Nigerian HEIs. The interviews were recorded and transcribed manually. In the e-interviews, unclear answers or those that will need further probing were probed through follow-up emails. Only one respondent did not respond to the probe. In the second stage, the evaluation of the framework, all respondents but three of the respondents provided feedback on the practicality of the framework via email (Figure 1). The method of analysis that was thematic but manually conducted is presented in the following section.

Thematic analysis with the aid of software, NVivo for Mac, was adopted. According to Dainty et al. (2000), the use of software analysis in QA helps in improving trustworthiness in that it ensures methodological transparency and reduces bias. Indeed, in the thematic analysis, the manifest and latent content can be explored which produces a visual presentation of themes, codes and their relationships 
(Vaismoradi et al. 2013). As purposeful sampling was adopted, the data analysis was conducted alongside the data collection. The preliminary data analysis started when collecting data from the first few participants, following the six phases of analysis i) Familiarisation with the data, ii) Initial codes, iii) Developing or searching for themes, iv) Reviewing themes, v) Defining and naming themes, and vi) Writing up in thematic analysis as noted in Braun and Clarke (2006).

The data analysis started with reading the data many times as the data was collected so as to be familiar with the data. During this process, the main investigator noted memos alongside those noted during the interviews. Before reading any interview, it was transcribed verbatim. This was followed by the second phase of the data analysis involving a deductive approach where the initial analysis is informed by the literature review conducted and the inductive approach. While the thematic analysis was conducted in the study, the analytical process slightly adopted what can be best described as some techniques or steps in grounded theory-open codes and axial codes (Struass \& Corbin 1990). While open coding helped organise the data into manageable chunks (Bowen 2008), the axial coding examined the interrelationships or connections among the codes. This procedure can be likened to 'grounded theory lite' (Braun \& Clarke 2006) where coding is akin to thematic analysis but not directed to theory development - 'full fat' grounded theory (Holloway \& Todres 2003 in Braun and Clarke 2006). However, the authors of the current paper view it as adopting a grounded theory 'lite' approach to data analysis (Heydarian 2016). This does not in any way affect the result of the data, but rather helped in data management.

While analysing the data, 'asking questions' - an analytical coding procedure (Basit 2010) - and constant comparisons were adopted, helping in triangulate the data and in exploring the meanings that emerged from the data. Example of the questions include what is happening here, what information is missing here. Furthermore, lineby-line coding was also conducted where the sentences were examined. This is where a lot of frequently used words by the respondents that contributed to the coding process at various stages were captured (Bowen 2008). Latent and manifest contents were also explored and contributed to the coding process. The matrix analysis feature of the NVivo for Mac contributed to the constant comparison. After this, the codes were reexamined to see if there are other codes that would emerge and to resolve coding disputes. Some of the codes that were consistent with each other were then developed into categories after which they were reexamined to see if they can be reduced or enhanced, or if there were any disputes to resolve. Many of the codes were noted as a memo or annotated. After this, the categories were distilled and then arranged into themes. This was the third phase. The fourth phase involved checking the themes against the dataset that is phase 1 and phase 2 (Braun \& Clarke 2006). At this stage, any theme that was similar to another was merged, and those that were not themes were removed. This led to the defining and naming of themes, ensuring that each theme tells a story about the data and is in line with the research questions (Braun \& Clarke 2006). After this stage, the results were presented with corresponding quotes where possible.

\section{RESULTS}

\section{Overview of the profile of the respondents}


The respondents were made up of non-research/lecturing students and academics from private and public Higher Education Institutions (HEIs) in Nigeria. All the respondents hold a first degree from a Nigerian HEI with a mix of Postgraduate qualifications such as Masters and PhD from both HEIs in Nigeria and abroad. Eight of the respondents have Doctorate degrees, while six have MSc or MPhil but are currently studying for a Doctorate and the remaining academics have only Masters degrees. Out of those that have doctorates, three held a postdoc position. These academies come from various departments in the Built Environment (BE) such as architecture, building, urban and regional development, estate management, civil engineering, construction management, but their teaching activities are multidisciplinary and cut across various departments within the BE. One student is a fresh first-degree graduate (having worked as a research assistant and a trainee statistician) and four were Masters' research students who have started their research projects. Their years of experience in academia also demonstrate acceptable levels of knowledge and experience required to answer the questions. The six geopolitical zones of Nigeria are well represented in the study. Although sampling students and academics without a doctorate training may skew the results as they may lack research training and skills, the following should be noted. First, six of the academics without a doctorate are currently studying for one but most importantly are research active, as research output counts towards the Continuous Professional Development (CPD), one of the requirements for promotion. Second, the four students were research students in Masters/PhD and MPhil programmes while the first-degree graduate has research experience. Third, the rationale for sampling the students was for triangulation. The authors view that the ability of the students to offer both students and (early or trainee) researcher perspectives would enrich and triangulate the data. It will also ensure that the data is not skewed to any particular level of research experience.

In the second stage of the research, the test for the practicality of the framework, 11 academics responded. All had doctorates in the following areas: Architecture, Civil Engineering, Construction Management, Estate Management, Housing and Urban development, and Project management. These were obtained in Nigeria and abroad. Of the respondents, two are professors, seven are senior lecturers and the rest lecturers, all in BE disciplines in private and public universities in Nigeria and abroad. Five of the respondents are female and the rest male. Factoring in the genders shows the genders that were represented as the problems is a social issue. It also ensures inclusiveness of all genders.

\section{Challenges in qualitative research within Built Environment Disciplines in Nigerian HEIs}

Almost all the respondents viewed issues relating to information as barriers to QA to research (Table 1) and stated that they do not view QA data as credible. Many of the respondents opine that the attitude towards providing information (see information constraints: Table 1) is as a result of the suspicious nature of the society (a cultural thing) and due to 'fear of the unknown'. Few respondents, including students, mentioned that research participants would require financial gratification before or after taking part in interviews. However, the opportunity presented by the attitude of the society towards 'interviews' offers some optimism (see Table 2). 
Table 1: Summary of challenges to qualitative research approach in Nigerian HEIs

\begin{tabular}{|c|c|}
\hline Themes & Evidence \\
\hline $\begin{array}{l}\text { Information } \\
\text { constraints }\end{array}$ & $\begin{array}{l}\text { Purposefully withholding information } \\
\text { Sceptical to provide information } \\
\text { The culture of deliberately providing misleading information } \\
\text { Unfavourable attitude of organisation to providing information } \\
\text { Lack of access to secondary data e.g. records. }\end{array}$ \\
\hline $\begin{array}{l}\text { - Social, cultural and } \\
\text { economic context } \\
\text { issues }\end{array}$ & $\begin{array}{l}\text { High level of illiteracy } \\
\text { Gender bias in the society } \\
\text { High level of poverty and insecurity in the society } \\
\text { Language barrier } \\
\text { Cultures in some communities that do not support research. } \\
\text { Populates' lack of understanding of the role of research. } \\
\text { Sceptical attitude towards research. }\end{array}$ \\
\hline - Logistical issues & $\begin{array}{l}\text { No access to analytical tools. } \\
\text { Unavailability of transcribers. } \\
\text { Lack of training on QA to research e.g. software training. } \\
\text { Language interpretation issues. } \\
\text { Difficulty in accessing data from organisations and participants } \\
\text { Lack of funding, person power, interpreter \& research instruments, and } \\
\text { ethical issues. }\end{array}$ \\
\hline $\begin{array}{l}\text { - Inadequate research } \\
\& \text { teaching focus and } \\
\text { motives }\end{array}$ & $\begin{array}{l}\text { No research direction in educational institutions } \\
\text { The dominance of passive teaching, at the expense of active teaching. } \\
\text { The wrong motive for research e.g. for promotion purposes. }\end{array}$ \\
\hline $\begin{array}{l}\text { - Attitude of the } \\
\text { industry and } \\
\text { academics towards } \\
\text { research }\end{array}$ & $\begin{array}{l}\text { Negative attitude of the construction industry towards research e.g. } \\
\text { practitioners resentment of academics. Lack of trust for academics. The } \\
\text { perception that organising QA is stressful and time-consuming e.g. collating, } \\
\text { transcribing and analysing the data. The unfavourable attitudes of senior } \\
\text { academics towards QA. Lack of understanding of the potential of QA to } \\
\text { research among academics e.g. that QA is unable to provide reliable data. }\end{array}$ \\
\hline
\end{tabular}

The social, cultural and economic contexts of Nigeria as highlighted in Table 1 remain significant as a challenge to QA research. Meanwhile, some of these contextual issues contribute to logistical issues. For instance, gender bias in the society-especially when it is cultural, traditionally or spiritually underpinnedmeans that the research will have to employ a male or female. Also, lack of trust by the respondents (in Table 1) means that a trusted person accompanies the investigator during data collection. This increases the cost of the research projects and questions the quality of the data. Meanwhile, more than four respondents pointed out gender issues as a significant barrier. Only one respondent noted that being a female was an advantage; to the other respondents, it is a disadvantage.

Regarding 'inadequate research and teaching focus and motives', four respondents stressed the lack of passion for research, emphasising that research is conducted for the wrong reasons. While two of the academics decried the untapped opportunities in Nigerian BE, one of the academic also noted the wrong motives for research and its implications. According to the respondent, 'The opportunities for qualitative research abound everywhere in the BE (in Nigerian HEIs)! In fact, we have not yet scratched the surface. Unfortunately, many academics view research as a means for promotion and not necessarily to generate new ideas or theories as should be. That is one of the main discouraging aspects of research here in Nigeria'. 
Further on 'inadequate research and teaching focus and motives', while three respondents demonstrated the passive nature of teaching in Nigeria's BE, one explicitly stated that: '...Nigerian academics are not open to progressive research methods. This (is) heavily supported by their indeed passive method of teaching. Switching from passive to participatory teaching is one way out'.

Equally important is the 'logistical issues' outlined in Table 1. The issues of transcribing and analysing data are emphasised by both academics and students, with some outlining the implications. One academics viewed that ' $\ldots$ the problem of qualitative research in Nigeria is the issue of transcribing and analysing it. We do not encourage it because we do not have the wherewithal to even transcribe it into sentences or the result that is desired... We do not really have ... the tools for analysing qualitative research in Nigeria'.

\section{Opportunities for qualitative research within Built Environment Disciplines in Nigerian HEIs}

Questions regarding the opportunities for QA to research within BE disciplines in Nigerian HEIs were posed and the responses support the comments expressed above by a respondent on the possibilities within BEs in Nigerian HEIs for QA research. This is where the respondent indicated that BE disciplines in Nigerian HEIs are a fertile ground for QA to research. One significant opportunity for QA research is that there is a realisation for a paradigm shift highlighted in Table 2 among a significant number of the respondents. One respondent stated: 'I think the time for qualitative research to be encouraged among all BE professionals is now since the widely used quantitative research (approach) has been unable to solve most of the issues affecting the BE significantly'.

Table 2: Summary of opportunities in qualitative research within the BE disciplines in Nigerian HEIs

\begin{tabular}{|c|c|c|}
\hline Themes & Subthemes & Evidence \\
\hline - Potentials of QA & Potentials of QA & $\begin{array}{l}\text { - The realisation for a paradigm shift } \\
\text { because of potentials of QA in } \\
\text { answering questions that quantitative } \\
\text { research cannot answer e.g. 'how' } \\
\text { and 'why' and some 'what' research } \\
\text { questions. }\end{array}$ \\
\hline - Contextual features & Characteristics of the $\mathrm{BE}$ & $\begin{array}{l}\text { - Complex scenarios in behavioural } \\
\text { patterns \& processes in Nigeria's BE. } \\
\text { - Nature of challenges in Nigeria's BE. } \\
\text { - Qualitative nature of architecture e.g. } \\
\text { areas of decoration, aesthetics. } \\
\text { - Higher level of illiteracy }\end{array}$ \\
\hline & $\begin{array}{l}\text { Characteristics of the } \\
\text { socio-cultural context }\end{array}$ & $\begin{array}{l}\text { The culture, history, and traditions of } \\
\text { developing countries. }\end{array}$ \\
\hline \multirow[t]{3}{*}{$\begin{array}{l}\text { - Conducive and } \\
\text { underexplored } \\
\text { environment }\end{array}$} & $\begin{array}{l}\text { Underexplored \& salient } \\
\text { areas }\end{array}$ & $\begin{array}{l}\text { - QA can help in exploring emerging } \\
\text { trends in Nigeria e.g. risk } \\
\text { management, gender issues QA. }\end{array}$ \\
\hline & & $\begin{array}{l}\text { - Respondents are more comfortable } \\
\text { discussing issues in interviews. }\end{array}$ \\
\hline & $\begin{array}{l}\text { Conducive environment } \\
\text { for trust \& relationship }\end{array}$ & $\begin{array}{l}\text { - Researcher gets to know and } \\
\text { understands the interviewee in QA. } \\
\text { - Familiarity between researcher and }\end{array}$ \\
\hline
\end{tabular}


respondents is improved.

- Commitment to QA in a quantitative dominant area.

The social and cultural contexts present opportunities because of the strong cultural sense and even social challenges such as the higher level of literacy. One respondent stated: 'When you look at our culture, our culture actually should favour qualitative research because Africans have a lot of history ... and people ordinarily would readily answer questions in depth rather than just answer sharp (closed) questions. Another respondent echoed this, saying that '...the African way of life is so rich and highly unexplored by Africans, in my opinion, especially lessons to be learnt regarding the $B E^{\prime}$.

While affirming the above and acknowledging the limitations of quantitative research in Nigerian HEI context, the response of another academic emphasised the ability of QA to research to obtain the 'hard to reach'. It also suggests that trust and a relationship can be established or improved between the respondent and the researcher. In particular, the respondent stated: 'From my experience I have learnt that using questionnaires to collect data from respondents is not reliable because most of them are not educated. They are more comfortable in discussing issues, as they perceive them because they feel their opinion counts'

\section{Ways to improving qualitative research within built environment disciplines in Nigerian HEIs}

Collaboration, reaching out calls for increased global exposure (Table 3): The idea here is to reach out to academics outside Nigerian HEIs and practitioners so as to learn from them. The reaching out will introduce academics in the Nigerian HEIs to what obtains outside Nigeria ensuring currency in academic activities. The respondents strongly opine that the views and ideas of people from abroad or the industry will 'inject' new ideas and attitudes.

Table 3: Summary of ways of improving qualitative research within BE disciplines in Nigerian HEIs

\begin{tabular}{lll}
\hline Themes & Subthemes & Evidence \\
\hline$\bullet$ Reaching out & $\bullet$ Collaboration & - Collaboration between academics in Nigeria and \\
& abroad on research projects, seminars, workshops \\
& and conferences. \\
& - Collaboration between academics and practitioners \\
& in research. \\
& - Partnership between academics in higher education \\
& institutions, research institutions and professional \\
& bodies in research. \\
& - Exchange programs between local and tertiary \\
& education institutions in developed countries. \\
& - Recruit foreign-trained academics to work in \\
& Nigeria. \\
& - Attend international programmes e.g. short courses. \\
\hline
\end{tabular}


- Self-exposure to global methods and practices by academics.

- Education and - Provide trainings awareness

- The role of academics

- Conducive environment
- Reorientation

- Increased awareness on QA

- Gradually introduce QA

- The role of senior academics

- Funding

- Journals as instruments for acceptance and adoption of QA
- Organising and hosting workshops, seminars, and conference on QA. Providing training on QA software.

- Research should be done for knowledge purposes and not for promotion

- Many academics called for increased awareness of QA, including its trustworthiness in the academia especially from those committed to QA. This can be through publishing quality QA paper in conferences and journals.

- Encourage QA through encouraging mixed methods research approach.

- Some of the respondents called for a shift in research paradigm from quantitative to QA

- Senior academics should encourage QA and not discourage it

- The change in research direction should come from the 'older hands'

- Senior academics should encourage covert research

- Increased research funding

- Establish QA alone journals in Nigeria

- Sensitise journals on QA

There are some relevant recommendations made by some respondents, for example triangulation as a way of gradually encouraging and introducing QA (Table 3). In particular, one respondent stated: 'Just to say that for the BE in Nigeria, we are still some way off from fully integrating qualitative research and perhaps the more gentle way to bring acceptability is to encourage triangulation and then from there gradually strive for acceptance of purely qualitative study'. The idea here is that while senior academics have roles to play, it should be gradual to ensure acceptance by academics. The senior academics also have a responsibility of driving change in HEIs research (Table 3).

Also, a respondent suggested 'covert research' as a way of tackling the challenges of QA to research within BE disciplines in Nigerian HEIs. According to the respondent, 'No doubt that we have recurring funding issues but the bureaucracy and logistics of setting up interviews is similar to what is obtained in the developed world. Because we have poor record keeping approaches and culture of deliberately giving misleading information, the 'fly on the wall' approach is what we need to move the industry forward at this stage, and we need to encourage our students to be research investigators who will lead'. A few respondents suggested that senior academics could encourage covert research.

The matrix-coding query between locally trained respondents and those with a combination of Nigerian and foreign educational backgrounds was conducted. This provides an indication of what these two respondent groups have to say about the solution to the under-representation of QA to research in Nigeria. It was found that many academics of full Nigerian academic background view that providing training on QA to research through conferences, seminars and workshops both nationally and 
internationally will help improve the use of QA in research. Also, they called for collaboration between industry practitioners and academics and between academics and their counterparts abroad. A few of the respondents also suggested triangulation, while those with Nigerian and developed country academic backgrounds emphasised the need for 'collaboration' in Table 3.

\section{IMPLICATIONS OF RESULTS FOR DCs AND DISCUSSION}

\section{Challenges in qualitative research within BE disciplines HEIs}

Inadequate research and teaching focus and motives emerged as a challenge to QA to research within BE disciplines in Nigerian HEIs, where research is not done to gain academic knowledge but for purposes of promotion. As a result, many academics go for the more convenient option, that is, quantitative research. There is evidence that this finding is also experienced in other DCs. For example, in Ghana, Adjei and Owusu-Ansah (2016) found that academics have various motives for publishing (including for job promotion).

While some logistical issues, for example, lack of funding is consistent with Maina (2005), gender bias, a challenge in the current study, is evidenced in the experience of Maina as an opportunity (2015). This is where, as a woman, she considered her gender as an advantage in research. Maina (2005) also reports cases of insecurity, which is consistent with this study. Various other challenges outlined in Table 1 are consistent with previous research, for example, Hurst et al. (2015).

The implications of these include calls for measures that will change the motives and attitudes of the academics towards research and measures that emphasise the impact of research to teaching or practice in DCs. Further implications of the challenges, for example lack of conducive environment, include the fact that academics in DCs may not be motivated to adopt QA in research and even introduce students to it. The implications are not limited to Nigeria but include other countries in the DCs.

\section{Opportunities for qualitative research within BE disciplines in HEIs}

It emerged in the study that the conducive environments that the contexts of Nigeria present improve and establish trust and good relationship between research participants (especially the illiterate ones) and the researcher in QA. This is because as the local researchers adopt observation or face-to-face interviews, they immerse themselves in the community; the illiterates in the society get to see the researchers as their own, they may not be sceptical to divulge information, as some level of trust will be established. This, in turn, improves the quality of the data and even encourages participation in research. The opportunities in the socio-cultural context of other DCs including Libya, Malaysia and Ghana for QA is reported in literature, for example, Kheni (2008) and Bubaker et al. (2005).

Regarding 'commitment to QA in a quantitative dominant area' under the subtheme 'Conducive environment for trust \& relationship' (Table 2), that academics take time to encourage students in QA, is not to say that it cannot occur in quantitative research teachings. Rather the point is that if academics can champion QA in a quantitative dominated area such as the BE, students may see the academics as educational models, and follow in their footsteps as academics leaders. This opportunity is significant, as Pryor (1998) has earlier suggested passive teaching as a challenge. 


\section{Ways to improve qualitative research within BE disciplines in HEIs}

The opportunities and recommendations by the respondents are covered in the section that follows. The matrix-coding query indicates possible propositions that may need further validation. In particular, the education background of academics in Nigeria is likely to determine their acceptability of measures for improving QA in BE research within HEIs.

\section{DEVELOPMENT OF THE QA IMPROVEMENT FRAMEWORK}

The overall comments of the 11 respondents on the practicality of the framework were positive. The majority of the respondents suggested improved ways of presenting the framework for better clarity including the input, outputs and various stages of the framework in the graphical presentation. As a result, a spider graphic presentation of the framework was dropped for the current graphic presentation.

\section{Overview of analytical framework}

Figure 2 shows the QA improvement framework developed using the results obtained in the study. However, in developing the framework, some key questions were asked. How will the challenges reported in this study be addressed? How can the opportunities in this study be exploited to the advantage of QA to research? How will the recommendations of the respondents be implemented? There are some keys points that must be observed in answering these questions. First, some of these challenges reported in this study are consistent with what obtains in developed countries and are generic. Second, some of these recommendations in Table 3 already address the challenges in Table 1, as some respondents have already provided detailed accounts of ways to improving the use of QA in BE research. Third, some of these opportunities that DCs present outweigh the challenges in Table 1. Based on the above, the framework is mainly built on the recommendations of the respondents towards addressing the challenges uncovered in this study, and then demonstrating how the opportunities will be exploited for improving QA in the BE research of Nigeria and possibly, other developing countries' HEI.

\section{The Framework}

Figure 2 is a graphic presentation of the framework, which comprises of the elements such as, 'need identification', 'collaboration', 'assessment of interim outcome and review of the treatment stage', 'preferential treatment of QA', 'increased research funding', 'regulatory mechanism' and 'increased level of awareness of QA'. These will need the input of, among many, academics, HEIs, the government, and companies. The elements are encapsulated in the five stages namely, 'preparatory', 'advocacy', 'treatment', 'interim outcome', and 'overall target'. 
Figure 2: A graphic presentation of the framework for improving the underrepresentation of QA in BE research in Nigeria HEIs

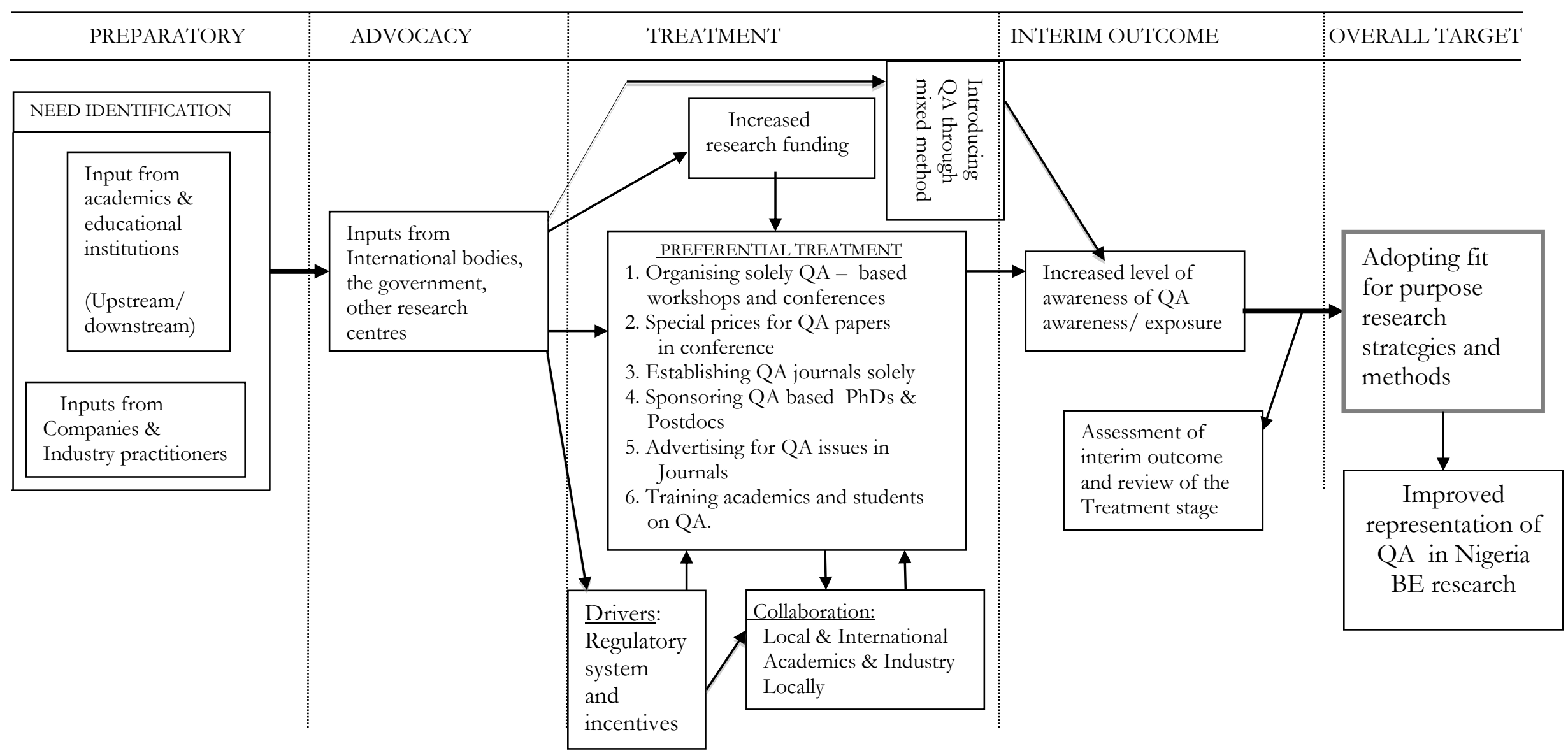





\section{Preparatory}

This is the first stage of the framework. The input all academics, HEIs and companies above are needed at this juncture. This 'need identification stage' involves the research, planning and agreements, setting the stage for the QA adoption improvement project. Equally important is the 'Upstream to the downstream system'. This is based on 'The role of senior academics' where they are expected to drive the change (Figure 2). It relates to one of the characteristics of Emerging Global Model (EGM), changes in the academic profession (Mohrman et al. 2008), which involves the improvement of QA through a hierarchical approach. EGM is discussed in details in 'collaboration'. The senior academics or 'those at the top of the academic affairs' are to drive the change, determining the direction of teaching and research in departments and faculties (see Mohrman et al. 2008). However, it can be argued that given the evidence in the current study that they have an antagonistic attitude towards QA to research, the workability of this is questioned. It should be noted that the findings of the present study do not show the proportion and influence position of the academics with the hostile attitude towards QA to research. Furthermore, the results indicate that some senior academics have a favourable attitude towards QA to research. Nonetheless, issues such as interpersonal conflicts, ownerships and copyright issues may have significant negative implications for the improvement of QA.

\section{Advocacy}

This is where the plans for improving research in the BE is put forward to the government with the help of the industry and most importantly international bodies. The government, international agencies, research centres and companies work towards the next stage, treatment.

\section{Treatment}

Here, there is a conscious effort - with possible implications of biased measures - to improve the adoption of QA in BE research. The strategies are discussed below.

Collaboration: Table 3 shows that much emphasis has been placed on 'collaboration' a subtheme in reaching out. This mainly comes in three ways. One way is the collaboration and association between academics and HEIs abroad and those in Nigeria (Figure 2). This can be through conferences, seminars and workshops. This should be done in such a way that it is within reach of HEIs in DCs such as Nigeria in that the host will be a Nigerian HEI. This collaboration will help local academics form relationships with their colleagues from abroad and collaborate on projects, addressing challenges in Table 1 such as and 'logistics issues'. The outcome of such a relationship will mean that the knowledge or experience gained will be transferred to students and even academics locally, addressing some issues in the themes in Table 1, the 'attitudes of the industry and academics towards research'. Countries such as China engage, promote and support collaboration in terms of research between local universities and other universities, laboratories and scholars in developed countries such as UK (Huang 2015). According to Huang (2015), this strategy has considerably improved the research in Chinese universities, making them attain world-class status. For example, Tsinghua University ranks 49th in the Times Higher Education ranking of 2014 (Postigglione \& Arimoto 2015). Other authors such as Mohrman, Ma, and Baker (2008) also discuss the role of international collaboration on research projects as one of the characteristics of the EGM, which has recorded success in improving research in universities. Mohrman et al. (2008: 21) describe EGM as 'an 
intensification and globalisation of the development of research universities in general'. Collaboration at this level (i.e. between local and international parties) will also lead to increased global exposure, which will also lead to the targetimprovement of the representation of QA in BE research in Nigerian HEIs. As stated elsewhere in this paper, academics should take advantage of IT and connect with their counterparts in developed countries. This will help increase the level of global exposure and will need the contribution of academics and educational institutions as delineated in the framework.

Although some international conferences such as the West African Built Environment Research (WABER) Conference, International Conference on Infrastructure Development in Africa (ICIDA) are already in place, providing the opportunities for academics and practitioners in various developing countries to integrate, there is still an opportunity for improvement. These conferences rotate the venues of their conferences, but these are limited to only partnering nations. It appears that academics have individual roles to play as well. Academics can exploit the opportunities provided by the Internet in that they can contact their colleagues aboard through social media.

The second way that the collaboration or association can occur is between academics and industry practitioners (Mohrman et al. 2008) (see 2 under collaboration in Figure 2 ). While conferences such as those above can be one of the ways through which both parties meet, it is possible that only practitioners who it is convenient for, or who are aware of it and passionate about research will attend. The findings suggest that academics should write to industry practitioners or approach them through networking or 'connection', as it is called in Nigeria. When academics undertake research into these firms with real life problems, or if these companies inform academics of their challenges and scholars tailor their research towards solving the problems, the relationship with and involvement of the company in the research may be possible. At the successful completion of the research project and when the problems outlined by the company are addressed, these companies may be more interested in commissioning further research. This can address some issues in the themes in Table 1, the 'attitudes of the industry and academics towards research' and information constraints'. Collaboration can also be between academics in BE and their colleagues in Arts or social science, the third way of collaboration.

Preferential treatment for qualitative research: This is informed by the concept of 'positive discrimination'. It is giving qualitative research a favourable or advantageous treatment (Figure 2). Themes that emerged and are outlined in Table 3 that fall into the context of 'Preferential treatment for qualitative research' include: paradigm shift from quantitative research to QA to research, providing training in QA to research, establishing QA to research only journals in developing countries such as Nigeria, and gradually introducing QA to research. Other measures that can be used for preferencing QA to research but not outlined in Table 3 include advertising for solely QA research funding, QA based doctoral sponsorship, allocating special prizes for best QA papers in workshops, conferences and seminars, which require inputs of academics and HEIs. The training of academics and students in QA can also be adopted here (Table 3). The points so far address challenges in Table 1 related to education and awareness, logistics, inadequate research focus and attitudes of academics towards research. The benefits of adopting research strategies and methods 
that are fit for purpose, by implication QA, can also be emphasised and covered in this training. Increasing the level of awareness is pivotal to the improvement of QA in Nigerian HEIs, as shown in Table 3. Authors such as Loosemore et al. (1996), Dainty et al. (1997, 2000), Carter \& Fortune (2004) have raised the awareness of the underrepresentation of QA in construction management in general. According to Carter and Fortune (2004), there has been an improvement in the adoption of QA in construction management research as a whole which is likely as a result. This does not only show the potential roles of academics in HEIs, but it also offers optimism for the success of the framework proposed.

Concerning increased research funding, the role of funding, especially from the government, is evidenced to have helped improved research and university ranking in China (Huang 2015). However, the contributions of charity organisations, international bodies, and companies, especially multinationals are pertinent in the adoption of the QA in research. These are adequately covered in the literature review

- Bernanke (2011), Maloney and Schumer (2010), Narayan (2010), and United Nations Educational, Scientific and Cultural Organization (UNESCO) (2015). However, the findings of the literature review that the private sector is not interested in basic research and mainly interested in applied research or research for commercial gain remains a concern. This raises the question of how the problem will be addressed in the context of Nigeria? The framework presented in Figure 2 shows that adequate funding will be required for preferential treatment of QA to research, but like every workshop, prize awards in conferences and other methods of motivation, funding is needed. This addresses challenges not limited to logistics issues.

Drivers: regulatory, and incentive mechanism. Although not covered in Table 3, there is the need for administration of rules and its control, drivers and incentive for selfcompliance. This can come in two ways. Firstly, there can be rules and regulations for academics by HEIs to address the bias towards QA, for example a penalty for academics that unfairly penalise students for adopting QA. This is akin to the research publications requirements for promotion noted elsewhere in this paper. Secondly, there can be incentives for adopting QA and all in the element, preferential treatment. An example can be if the publication requirement for CPD or promotion is two, one QA paper should be an equivalent. However, this should be an interim measure so as to avoid over-adopting QA to research, a bias against quantitative strategies or a neglect of research problems that quantitative strategies should address.

\section{Interim outcome and overall Target}

Increased level of awareness on $Q A$ is the interim outcomes but will be assessed; if the outcome is unexpected, the treatment stage will be reviewed. The overall target is the adoption of fit for purpose research strategies and methods by implication, the improved representation of QA in Nigeria's BE research.

\section{The potentials of the framework in the midst of the opportunities provided by qualitative research in Nigerian HEIs}

While it is established that there are opportunities in Nigerian HEIs for QA, it is vital to indicate the potentials of the developed framework in the midst of the possibilities. First, preferential treatment for qualitative research will be encouraged, but the drivers and increased funding in Figure 2 and the 'realisation for a paradigm shift' and the 
'underexplored and salient areas' in Table 2 may also encourage its adoption. Secondly, all statements highlighted in Table 2 will make collaboration succeed. For instance, commitment to QA to research in quantitative dominant, underexplored and salient areas, socio-cultural contexts will encourage and attract partners in developed countries or the industry.

\section{Addressing the challenges to the adoption of qualitative research approach in Nigerian HEIs}

The challenges to the adoption of QA in Nigerian HEIs itemised in Table 1 such as 'time constraint', 'inadequate research and teaching focus and motive', 'logistical issues and poor understanding of QA' can be addressed by improving the level of awareness to qualitative research. This is because the adequate knowledge of the research method is generic with QA to research and is consistent with its adoption in developed countries. Further, exposure to emerging data collection methods in QA to research such as e-interviews will also help as it reduces the time for transcription and analysis. This is in addition to establishing informality between the interrogators and the interviewee (Bampton \& Cowton 2002), thus improving the level of trust.

\section{CONCLUSIONS}

Using Nigeria as a case, the study examined the challenges to and opportunities for establishing QA to BE research in HEIs and developed an improvement framework for QA. While no claim is made that this paper advances the development of qualitative methods, it contributes to the extant literature on the challenges that researchers in developing countries face regarding the adoption of QA to research. Importantly, the study argues that research methods or strategies adopted should be 'fit for purpose.'

It emerged that the challenges in $\mathrm{BE}$ encompass inadequate research teaching and motives, information constraints, the contexts of DCs, among other things, logistical issues. The opportunities in DCs and the BE discipline can support QA and measures to improve the adoption of QA in DCs' HEIs. These opportunities are not limited to the: realisation for a paradigm shift; contextual features (characteristics of the sociocultural context, and features of BE); underexplored and salient areas and general potentials of QA. These opportunities will support improvement measures such as collaboration at various levels, gradually introducing QA through triangulation, encouraging passion driven teaching and research.

The proposed improvement framework for QA in BE research encompasses: preferential treatment for QA to research; collaboration between academics in developed countries and Nigeria, between scholars and practitioners; and drivers: regulatory, and incentive mechanism. There are also provisions for evaluation and reviewing of the framework. The framework is practical, specifying the roles that academics can play as individuals and the roles of the academia as an institution.

The value and implications of the research for other countries including DCs are not limited to the following. The awareness of BE researchers and academics of the challenges and opportunities in QA and academics how they knowingly or unknowingly hinder the research is demonstrated. Academics (based locally and 
internationally), policymakers, and the government can adopt the framework, at least as a stepping stone, to improving the adoption of adequate research strategies and methods that are fit for purpose, by implication including QA. Also, the training and teaching of QA can be informed by a good understanding of contextual issues and opportunities and these should also be included in the content of the research methods modules. By implication, the research will be aligned to addressing the challenges in the society including research problems, underpinned by a good understanding and consideration of the contexts of the country but most importantly offer practical recommendations. The study serves as a contrasting standard to countries where QA is adequately adopted and makes a case for the continuity.

Some limitations of this study include the lack of government perspective in the study, a key stakeholder in research, which may have implications for the results; therefore, further studies are recommended to address this. Additionally, because this framework was not tested in a real life situation, some of its parts may need to be revised. However, as the framework is mostly based on responses provided by stakeholders in academia and its practicality assessed by academics, a case is made for its workability. Further studies can also seek to validate possible propositions in this study, such as the influence of educational background/discipline of academics on their acceptance of measures proposed for improving QA within BE research in HEIs.

\section{REFERENCES}

Adjei, K. O. K., and Owusu-Ansah, C. M. (2016) Publishing preferences among academic researchers: implications for academic quality and innovation. Library Philosophy and Practice (ejournal). 1349,1-15.

AlSehaimi, A., Koskela, L., and Tzortzopolulos, P. (2013) Need for alternative research approaches in construction management: case of delay studies. Journal of Management in Engineering, 29 (4), 407-413. Doi 10.1061/(ASCE)ME.1943$\underline{5479.0000148}$

Bampton, R. and Cowton, C. J. (2002) The e-Interview forum qualitative, Sozialforschung/Forum: Qualitative Social Research, 3 (2) Art 9. Retrieved on 0301- 16 http://www.qualitative-research.net/index.php/fqs/article/view/848/1843

Basit, T. (2003) Manual or Electric? The role of coding in qualitative data analysis, Educational Research, 45(2), 143- 154.

Bernanke, B. S. (2011) Promoting Research and Development The Government's

Role. Issues in Science and Technology 27, no. 4 (Summer 2011)

Bowen, G. A. (2008) Naturalistic inquiry and the saturation concept: a research note. Qualitative Research, 8(1), 137 -152

Braun, V. and Clarke, V. (2006) Using thematic analysis in psychology, Qualitative Research in Psychology, 3:2, 77-101

Bubaker, S., Balakrishnan, P. and Bernadine, C. (2005) Qualitative case study research in Africa and Asia: Challenges and prospects. Procs. 3rd International Qualitative Research Convention, Malaysia, 1-13.

Carter, K. and Fortune, C. (2004) Issues with data collection methods in construction management research. In: Khosrowshahi, F (Ed.), Proceedings 20th Annual ARCOM Conference, 1-3 September 2004, Edinburgh, UK, (2), 939-46.

Castillo-Montoya, M. (2016) Preparing for Interview Research: The Interview Protocol Refinement Framework. The Qualitative Report, 21(5) 811-831. 
Creswell, J. W. (1998) Qualitative inquiry and research design; choosing among five traditions. California: Sage Publishing.

Crossley, M. and Vulliamy, G. (1996) Issues and trends in qualitative research: Potential for developing countries. International Journal of Educational Development, 16(4), 439- 448. Retrieved from doi:10.1016/S0738-0593(96)00064$\underline{8}$

Dainty, A. R. J., Bagilhole, B. M., and Neale, R. H. (1997) Analytical Strategies for dealing with qualitative data in construction management research. In Stephenson, P. (Ed) Proceedings 13th Annual ARCOM Conference, 15 - 17 September 1997, King's College, Cambridge, (2), 484 - 493.

Dainty, A. R. J., Bagilhole, B. M., and Neale, R. H. (2000) Computer aided analysis of qualitative data in construction management research. Building Research and Information 28(4), 226-233. DOI: 10.1080/09613210050073689

Denzin, N. K. (1970). The research act: A theoretical introduction to sociological methods. Chicago: Aldine Publishing Company

Ejohwomu, O. A. and Oshodi, O. S. (2014) A review of construction management and economics research outputs in Nigeria: To towards a sustainable future.

Journal of Construction Project Management and Innovation, 4, (S1), 900 - 905.

Retrieved from

http://reference.sabinet.co.za/webx/access/electronic_journals/jcpmi/jcpmi_v4_sup p1_a2.pdf

Halai, N. (2011) How teachers become action researchers in Pakistan: emerging patterns from a qualitative meta-synthesis. Educational Action Research, 19(2), 201-214. DOI: 10.1080/09650792.2011.569213

Heydarian, N. M. (2016) Student Handbook: Developing theory with the ground theory-approach and thematic analysis. Observer, 29(4). Retrieved on 26-09-16 from http://www.psychologicalscience.org/index.php/publications/observer/2016/ april-16/developing-theory-with-the-grounded-theory-approach-and-thematicanalysis.html

Huang, F. (2015) Building the world-class research universities: a case study of China. Higher Education 70(2), 203-215. DOI: 10.1007/s10734-015-9876-8

Hughes, W. (2010) Built Environment education, research and practice: Integrating diverse interests to make an impact. In: Laryea, S., Leiringer, R. and Hughes, W. (Eds) Procs West Africa Built Environment Research (WABER) Conference, 27-28 July, Accra, Ghana, 1-8.

Hurst, S., Arulogun, O. S, Owolabi, M. O., Akinyemi, R., Uvere, E., Warth, S. and Ovbiagele, B. (2015). Protesting Qualitative data collection Procedures to facilitate Methodological Adherence and Team building in Nigeria. International Journal of Qualitative Methods, 14(1), 53 - 64. Retrieved from https://ejournals.library.ualberta.ca/index.php/IJQM/article/view/21849/17922

Hyari, H.K., and El-Mashaleh, M.S., and Rababeh, S.M. (2015). Framework For Managing The Traffic Impacts of Building Construction Project. Journal of Construction in Developing Countries, 20(2), 97 - 113. Retrieved from http://web.usm.my/jcdc/vol20_2_2015/JCDC\%2020(2)\%202015-Art.\%206(97113).pdf

Isaacs, A. N. (2007). Strengthening research in community medicine. Indian Journal of Community Medicine, 32(4), 239- 240. DOI: 10.4103/2141-9248.144889

Isaacs, A. N. (2014) An overview of qualitative research methodology for public health researchers. International Journal of Medicine and Public Health, 4(4), 318- 323. DOI: 10.4103/2230-8598.144055 
Kheni, N. A. (2008). Impact of health and safety management on safety performance of small and medium-sized construction business in Ghana (Doctoral Thesis Loughborough University). Retrieved from https://dspace.lboro.ac.uk

Laryea, S., Leiringer, R. (2012) Built Environment research in West Africa: current trend and future direction. In: Laryea, S., Agyepong, S. A., Leiringer, R. and Hughes, W. (Eds) Procs $4^{\text {th }}$ West Africa Built Environment Research (WABER) Conference, 24 - 26 July, Abuja, Nigeria, 797 - 804.

Loosemore, M. Hall, K and Dainty, A. (1996) Innovation and courage in construction management research, Procs., $12^{\text {th }}$ Annual ARCOM Conference, $418-427$.

Maina, J. J. (2015) Architects and interdisciplinary research: Reflections from ethnographic and measured fieldwork. In: Laryea, S. and Leiringer R. (Eds) Procs $6^{\text {th }}$ West Africa Built Environment Research (WABER) Conference, 10-12 August, Accra, $131-145$.

Maloney, C. B., and Schumer, C. E. (2010) The pivotal role of government investment in basic research. Final RD Report, United States Congress Joint Economic Committee. Retrieved on 19-04-17 from https://www.jec.senate.gov/public/index.cfm/democrats/reports1?ID=D6E234671767-40E4-9481-3990F5F93C61.

Mohrman, K., Ma, W. H., \& Baker, D. (2008). The research university in transition: The emerging global model. Higher Education Policy, 21, 5-27. Retrieved from doi:10.1057/palgrave.hep.8300175

Morgan, D. L. (2006) Practical Strategies for Combining Quantitative and Quantitative Methods: Applications to Health Research. In: Emergent Methods in Social Research (edited by Hesse-Biber, S. N. and Leavy, P.). Sage Publications Inc., London, 165-182.

Mugisha, J., Knizek, B. L., Kinyanda, E., and Hjelmeland, H. (2011). Doing Qualitative Research on Suicide in a Developing Country: Practical and Ethical Challenges. Crisis, 32(1),15-23. DOI: 10.1027/0227-5910/a000047

Narayan, A. K. (2012) The role of government and accounting in the development of academic research commercialisation: The New Zealand experience. Accounting History, 17 (3-4), 311-329.

O'Reilly, M. and Parker, N. (2012) 'Unsatisfactory Saturation': a critical exploration of the notion of saturated sample sizes in qualitative research. Qualitative research, 12(2), 190-197.

Panas, A. and Pantouvakis, J. P. (2010) Evaluating research methodology in construction productivity studies. The Built and Human Environment Review, 3(SP.1) 63- $65 . \quad$ Retrieved from http://citeseerx.ist.psu.edu/viewdoc/download?doi=10.1.1.403.4785\&rep=rep1\&ty pe $=$ pdf

Patton, M. (1990) Qualitative evaluation and research methods (169-186). Beverly Hills, CA: Sage.

Postiglione, G. A. and Arimoto, A. (2015) Building research universities in East Asia. Higher Education (70), 151-153. DOI: 10.1007/s10734-015-9873-y

Pryor, J. (1998). Action research in West African schools: Problems and Prospects. International Journal of Educational Development, 18(3), 219 -228. doi:10.1016/S0738-0593(98)00020-0

Saunders, M., Lewis, P. \& Thornhill, A., (2009) Research Methods for Business Students (5th ed.), London: Prentice Hall.

Strauss and Corbin (1990). Basics of qualitative research: Grounded Theory Procedures and Techniques. London: Sage. 
Tertiary Education Trust Fund (Establishment, etc.) ACT, 2011, The Federal Government Printer, Lagos, Nigeria.

Umeokafor, N. (2015). A discussion of obser-view as a method of generating data in the construction industry. Civil Engineering Dimension, (Dimensi Teknik Sipil) 17(1), 54-58. doi: $10.9744 /$ ced.17.1.54-58

Umeokafor, N. I. and Windapo, A. O (2016) A framework for managing contextual influence on health and safety on construction projects. In Windapo, A.O, Odediran, S. J. and Adediran, A.; Proc. $9^{\text {th }}$ Construction Industry Development Board (CIDB) postgraduate conference, Cape Town, 2- 4 Feb. 2016, pp 285 - 294.

Umeokafor, N. I. and Windapo, A. O. (2017) Understanding the underrepresentation of qualitative research approaches to built environment research in Nigeria.

International Journal of Construction Education and Research.

dx.doi.org/10.1080/15578771.2017.1316799

United Nations Educational, Scientific and Cultural Organization (UNESCO) (2015) Science Report Towards 2030, UNESCO Publishing. Retrieved on 19-04-17 from http://unesdoc.unesco.org/images/0023/002354/235406e.pdf

Vaismoradi, M., Turnnen, H. and Bondas, T. (2013) Content analysis and thematic analysis: implications for conducting a qualitative descriptive study. Nursing and Health Science, 15, 398-405. 
